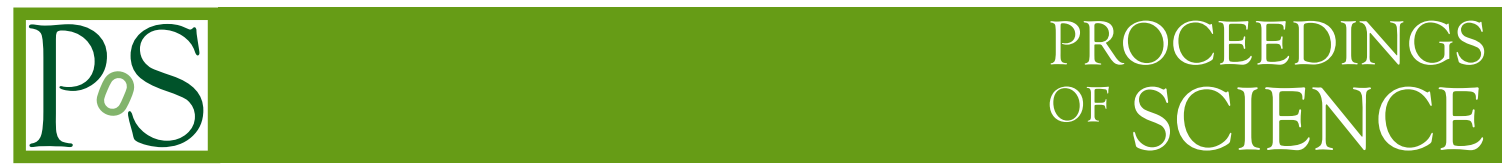

\title{
3D Pixels - recent results
}

E. Bolle ${ }^{9}$, M. Borri ${ }^{12}$, M. Boscardin ${ }^{15}$, G.-F. Dalla Betta ${ }^{14}$, G. Darbo ${ }^{13}$, C. Da Vià ${ }^{7}$, O. Dorholt ${ }^{9}$, S. Fazio ${ }^{3}$, C. Gemme ${ }^{13}$, H. Gjersdal ${ }^{9}$, P. Grenier ${ }^{10}$, S. Grinstein ${ }^{6}$, P. Hansson ${ }^{10}$, J. Hasi ${ }^{10}$, F. Huegging ${ }^{2}$, P. Jackson ${ }^{10}$, C. Kenney ${ }^{10}$, M. Kocian ${ }^{10}$, A. La Rosa ${ }^{4}$, A. Mastroberardino ${ }^{3}$, P. Nordahl ${ }^{9}$, F. Rivero ${ }^{12}$, O. Røhne ${ }^{* 9}$, H. Sandaker ${ }^{1}$, K. Sjøbæk ${ }^{9}$, T. Slaviec ${ }^{5}$, D. Su ${ }^{10}$, J. Tsung ${ }^{2}$, D. Tsybychev ${ }^{11}$, N. Wermes ${ }^{2}$ and C. Young ${ }^{10}$

${ }^{1}$ University of Bergen

${ }^{2}$ Bonn University

${ }^{3}$ Calabria University

${ }^{4}$ CERN

${ }^{5}$ Czech Technical University

${ }^{6}$ IFIC Barcelona

${ }^{7}$ University of Manchester

${ }^{8}$ University of New Mexico

${ }^{9}$ University of Oslo

${ }^{10}$ SLAC

${ }^{11}$ Stony Brook University

${ }^{12}$ Torino University

${ }^{13}$ INFN Genova

${ }^{14}$ Università di Trento and INFN Trento

${ }^{15}$ FBK Trento

Full-3D and Double-sided Double Type Column (DDTC) 3D Pixel detectors, bump bonded to the FEI3 read-out ASIC, has been characterized in a 1.6 T magnetic field using high-energy pions. We show pixel cell hit efficiency and charge sharing maps, and present first results on cluster size and track resolution, for normal and inclined tracks. The tracking performance of the 3D sensors is observed to be largely unaffected by the magnetic field.

VERTEX 2009 (18th workshop) - VERTEX 2009

September 13 - 182009

Veluwe, the Netherlands

* Speaker. 


\section{Radiation hard 3D Pixel sensors}

The combination of semiconductor sensor processing with deep reactive ion etch (DRIE) methods, as used for manufacturing of micro electro-mechanical systems (MEMS), allows for the fabrication of Silicon Pixel sensors where the electrodes are implemented as vertically etched columns, fully or partially penetrating the Silicon bulk [1] [2]. The general principle applied to a wide-pixel geometry is illustrated in Figure 1 Contrasting with planar sensors, the 3D architecture eliminates the need for guard ring structures and their associated edge-efficiency loss. The horizontal electrical field is terminated into an etched and doped edge-electrode, resulting in a sensor that is fully efficient all the way to the sensor edge [3] [4]. The penalty is that the volume occupied by the electrode columns is not fully efficient for tracks of normal incidence. The amount of this partially inefficient volume is determined by the electrode density and the hole aspect ratio; which for modern DRIE technologies can exceed 25.

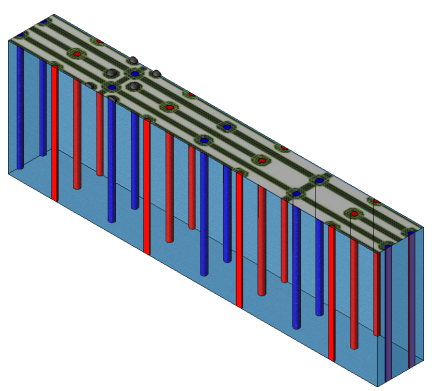

(a) Full-3D

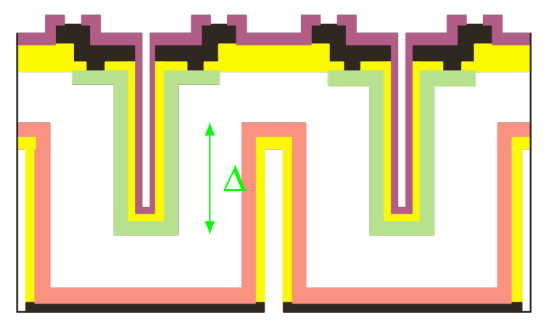

(b) DDTC
$103 \mu \mathrm{m}$

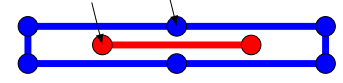

$71 \mu m$

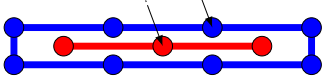

$564 m$

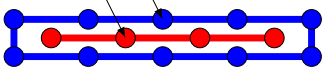

(c) Layouts

Figure 1: (a) Full-3D pixel sensor with embedded 3D electrodes. A $2 \times 2$ pixel mask detail is superimposed to indicate the cell geometry, here with 2 read-out electrodes (red) distributed along the pixel center and a corresponding number of bias electrodes (blue) along the long pixel edges, shared between neighboring pixels. (b) Sketch of double sided double type column (DDTC) sensor cross-section. The column overlap $(\Delta)$ is indicated as the vertical distance between the bottom of the read-out and the top of the bias columns.

(c) Pixel layout showing configurations with 2, 3 and 4 read-out electrodes per pixel.

The 3D electrode topology effectively decouples the drift distance from the track traversal thickness, and has a higher average electrical field at full depletion, compared to planar sensors. The resulting shorter signal collection time means that the trapping probability decrease. As the faster signal is achieved while maintaining sensor thickness, and hence the total charge, the results is greatly improved radiation hardness [5] [6] [7]. The decoupling of drift distance from the traversal thickness allows a certain degree of tailoring of sensor layout to detector requirements: Fewer read-out electrodes gives higher efficiency while shorter electrode distance implies more radiation tolerance.

The high electrode density and short electrode distance inevitably leads to increased detector capacitance, with the main contribution being proportional to the sensor thickness. For a thickness of $200 \mu \mathrm{m}$ the input-to-bias capacitance is significant, again contrasting with similar comparable planar pixel sensors, where the detector capacitance is low and dominated by the inter-pixel capacitance. Consequently, the 3D devices will have higher noise and larger time walk, and the 
minimum attainable in-time threshold will be higher, somewhat offsetting the benefits of having a higher signal after irradiation. Still, as has been shown in eg [8], 3D sensor designs can achieve adequate noise levels, even when operated with standard, non-optimized front-end electronics. It is also expected that some increase in the time walk can be offset by allowing for an increased input transistor current.

\subsection{Magnetic field in 3D sensor structures}

The effects of a magnetic field on the signal formation in 3D sensor structures are expected to be quite different from the well-known shifting and, in general, widening of the charge clusters seen in planar sensors. The electrical field is constrained to the sensor lateral plane. Assuming a barrel-type detector embedded in an axial solenoidal field, the action of the Lorentz effect will be to deflect the drifting charges out of the lateral plane. Since the projection of the drift is unchanged, no net effect on the cluster shape or position is expected. In a more detailed study one could expect to see an increase in the total signal collection time, and possibly some loss of signal as charges close to the surface are deflected into the oxide.

\section{Devices under test}

The sensors studied below are fabricated to be compatible with the ATLAS pixel geometry, having 18 columns by 160 rows of $50 \times 400 \mu \mathrm{m}^{2}$ pixels. All tested 3D samples were 3 -electrode designs and operated well above their respective full depletion voltages, as determined by the noise versus bias scan reaching the plateau. For reference, a single-chip size planar sensor of the standard ATLAS type was operated at a bias of $150 \mathrm{~V}$, labeled below as PLANAR.

\subsection{Hybridized detector assemblies}

Each test sensor was bump-bonded to ASICs identical to the front-end used by the current ATLAS pixel detector [9]. The FEI3 [10] is a versatile pixel read-out chip implemented in $0.25 \mu \mathrm{m}$ CMOS technology, reading out 2880 pixels organized in $9 \times 2$ columns of 160 rows. Apart from LHC-style trigger latency data buffering and associated time-stamping, the chip provides timeover-threshold based signal amplitude digitization, with per-pixel tunable thresholds and amplitude scale. For the present measurements the ASIC was clocked and read-out at the standard $40 \mathrm{MHz}$ rate, and the built-in signal injection system was used to tune the thresholds of each Device-underTest to $3200 \mathrm{e}^{-}$with a typical dispersion of $50 \mathrm{e}^{-}$. The charge digitization scale was set to 60 counts for $20 \mathrm{ke}^{-}$, the scale was cross-checked using a gamma source.

\subsection{Stanford Full-3D with active edge}

Full-3D sensors with various geometries have been pioneered at Stanford (CA, USA), see References [3] and [4]. The Stanford process requires that the active wafer is initially bonded to a mechanical support wafer, and all subsequent processing steps happen from the top side. There are separate etch-steps for the read-out and bias electrodes, one set of electrodes are doped and filled with poly-silicon before the other set is etched. The p-type trench electrode, defining the edge of the active area, is manufactured together with the bias electrodes. In a final step a metal layer is 
patterned, connecting the read-out electrodes for one pixel to its bump-bond pad, and adds a bias grid for the bias electrodes.

The batch used for the current tests was processed on $210 \mu \mathrm{m}$ thick wafers p-type wafers, with a theoretical full-depletion voltage of $15 \mathrm{~V}$ for the 3-electrode configuration. The tested sample is labeled STA-3EG below, and it was operated at a bias of $35 \mathrm{~V}$.

\subsection{FBK-irst Double-sided double type column}

The sensors from FBK-irst (Italy) [11] are fabricated in a double-sided process with one type of columns etched from each side, and the electrodes do not fully penetrate the bulk. The column electrodes are doped but remain unfilled, and there is no edge electrode. A front-side patterned metal layer connects the read-out electrodes to the bump-bond pads, the bias electrodes are connected to a back-side solid metal layer.

The two 3D-DDTC devices tested both had 3 read-out electrodes per pixel and were manufactured on $(220 \pm 10) \mu \mathrm{m}$ thick p-type wafers but originated from different batches. For the first batch the DRIE was performed externally, the column overlap was $90-100 \mu \mathrm{m}$ and the full-depletion voltage was $12 \mathrm{~V}$ for the 3-electrode geometry. During data taking it was biased at $35 \mathrm{~V}$. For the second batch FBK-irst did the DRIE step in-house; here the column overlap was $110-150 \mu \mathrm{m}$ and the full-depletion voltage was $4 \mathrm{~V}$. Due to leakage problems, this sampled could not be operated above $8 \mathrm{~V}$. The two samples are labeled $F B K-3 E M 5$ and $F B K-3 E 7$ respectively.

\section{Experimental setup}

The experimental set-up is largely similar to what has been used for earlier measurements of 3D Pixel detectors by a partially overlapping ATLAS-based group [12]. A major novelty is the use of an experimental magnet, presumed to be the first characterization of 3D sensors in a magnetic field.

\subsection{Particle beam, magnetic field and scintillator triggers}

The measurements were performed on the H8 beam line in the CERN North Area, using $180 \mathrm{GeV} / \mathrm{c} \pi^{+}$from a SPS secondary target. The experimental setup was mounted inside the bore of the "Morpurgo"-magnet, a superconducting dipole magnet that was recommissioned for the purpose of these measurements. The dipole provided a vertical magnetic field of about $1.6 \mathrm{~T}$ at the nominal current of $5 \mathrm{kA}$.

Two overlapping scintillators in front of the tracking system were used to provide a coincidence trigger; in the rear a large $150 \times 150 \mathrm{~mm}^{2}$ paddle scintillator with a $15 \mathrm{~mm} \varnothing$ hole was used in anti-coincidence mode, allowing efficient suppression of showers and multiple-scatter events. The veto was implemented purely for data-taking efficiency reasons, the final analysis only use successfully reconstructed tracks from clean events.

\subsection{Charged particle tracking and alignment}

Reference tracks were provided by three planes of double-sided silicon strip sensors, the Bonn ATLAS Telescope [13]. The telescope planes were mounted on a precision table together with the 
Devices-under-Test as illustrated in Figure 2a ; a single plane in front separated by about $900 \mathrm{~mm}$ from a pair of planes in the back. The doublet planes were mounted $60 \mathrm{~mm}$ apart.

After masking noisy strips, events with exactly one hit in each telescope plane were selected for reconstruction. The resulting beam shape is a convolution of the trigger and tracking efficiencies, shown in Figure 2. Tracks were fitted to a constant curvature helix, essentially treating all particles as having identical momenta, and the magnetic field as being uniform. Any model error arising from the actual field non-uniformity was absorbed in the transverse alignment, which was done independently for runs with and without a magnetic field. Excess extrapolation errors arising from a finite spectral width are expected to give a limited contribution to the tracking error.

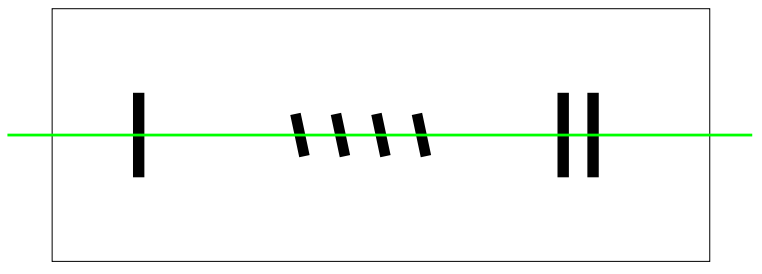

(a) Tracking setup

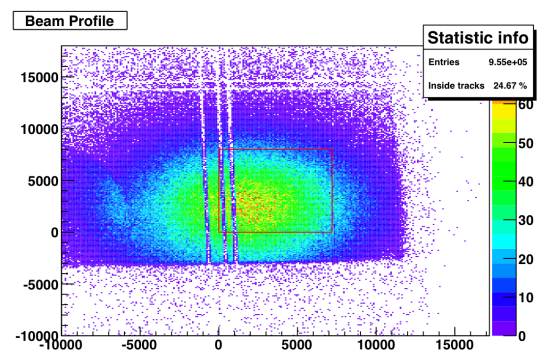

(b) Effective beamspot

Figure 2: Left: Tracking detectors on precision table, view from top. Right: Beam shape including trigger and reconstruction efficiencies. The horizontal and vertical shades are due to masked noisy strips in the telescope planes. The outline rectangle represents the acceptance of a $7.2 \times 8.0 \mathrm{~mm}^{2}$ test sensor.

The point resolution of the telescope was estimated comparing hits in one of the doublet planes to tracks extrapolated from the two remaining planes. The distribution of unbiased residuals is compatible with a toy Monte-Carlo model which assumes a point resolution of $5 \mu \mathrm{m}$. The same model predicts the error of the extrapolated track position in the Device-under-Test planes to be of order $3 \mu \mathrm{m}$, which we consider a lower bound on the total track resolution.

\section{Data taking and analysis}

A total of about 6 million triggers were recorded during the beam period in May/June 2009. Of these about 1.4 million events contained a single reconstructed track which passed through the Devices-under-Test. Roughly equal amounts of data were taken with the magnetic field on and off, and for each magnet setting a fraction data was taken with normal incidence as well as with inclined tracks.

The sensors tilted axis was parallel to the long pixel edge, the tilt angles were set by hand to approximately $15^{\circ}$ and the actual incidence angle of each sensor was recovered as part of the alignment procedure. Since all tracks are essentially parallel, a possible skew deformation of the entire telescope system is not constrained by the data. This limits the accuracy of the reconstructed incidence angle to about $0.4^{\circ}$.

The numerical results for hit efficiency, charge sharing probability, and point resolution are summarized in Table 1 An immediate observation is that all 3D sensors types recovers full efficiency for inclined tracks; this result is not affected by the magnetic field. A more detailed discussion of the of the various quantities are given in the following sections. 


\begin{tabular}{|c|c|c|c|c|c|c|c|}
\hline \multirow{2}{*}{\multicolumn{2}{|c|}{ Sensor and angle }} & \multicolumn{2}{|c|}{ Hit efficiency $[\%]$} & \multicolumn{2}{|c|}{ Charge sharing $[\%]$} & \multicolumn{2}{|c|}{ Resolution $[\mu \mathrm{m}]$} \\
\hline & & $B=0 \mathrm{~T}$ & $B=1.6 \mathrm{~T}$ & $B=0 \mathrm{~T}$ & $B=1.6 \mathrm{~T}$ & $B=0 \mathrm{~T}$ & $B=1.6 \mathrm{~T}$ \\
\hline \multirow[b]{2}{*}{ Planar } & $\phi=0^{\circ}$ & 99.9 & 100.0 & 20.4 & 45.4 & 13.8 & 10.2 \\
\hline & $\phi=13.8^{\circ}$ & 100.0 & 100.0 & 78.9 & 42.6 & 9.7 & 10.4 \\
\hline \multirow[b]{2}{*}{ STA-3EG } & $\phi=0^{\circ}$ & 96.7 & 96.5 & 20.2 & 23.0 & 14.3 & 13.9 \\
\hline & $\phi=11.4^{\circ}$ & 99.9 & 99.9 & 60.3 & 55.7 & 10.8 & 9.8 \\
\hline \multirow[b]{2}{*}{ FBK-3EM5 } & $\phi=0^{\circ}$ & 98.4 & 98.3 & 12.2 & 14.6 & 15.4 & 14.8 \\
\hline & $\phi=14.1^{\circ}$ & 99.8 & 99.5 & 62.1 & 52.5 & 11.9 & 11.3 \\
\hline \multirow[b]{2}{*}{ FBK-3E7 } & $\phi=0^{\circ}$ & 99.2 & 99.1 & 17.6 & 18.9 & 14.0 & 13.5 \\
\hline & $\phi=12.0^{\circ}$ & 99.8 & 99.9 & 51.6 & 47.1 & 10.4 & 9.7 \\
\hline
\end{tabular}

Table 1: Key performance results for operation with and without a magnetic field, using normal incidence as well as inclined tracks.

\subsection{Pixel efficiency}

The over-all hit efficiency is evaluated by extrapolating the tracks to the Device-under-Test plane and searching for hits in a $3 \times 5$ pixel $\left(1200 \times 250 \mu \mathrm{m}^{2}\right)$ region around the track. The efficiency loss in the 3D-devices at normal incidence clearly comes from the electrode regions, as shown in Figure 3. The fully-functional DDTC specimen ( $F B K-3 E 7)$ showing only a $1 \%$ loss indicates that the charge deposited in the bulk material above and below the column electrodes is collected with good efficiency.
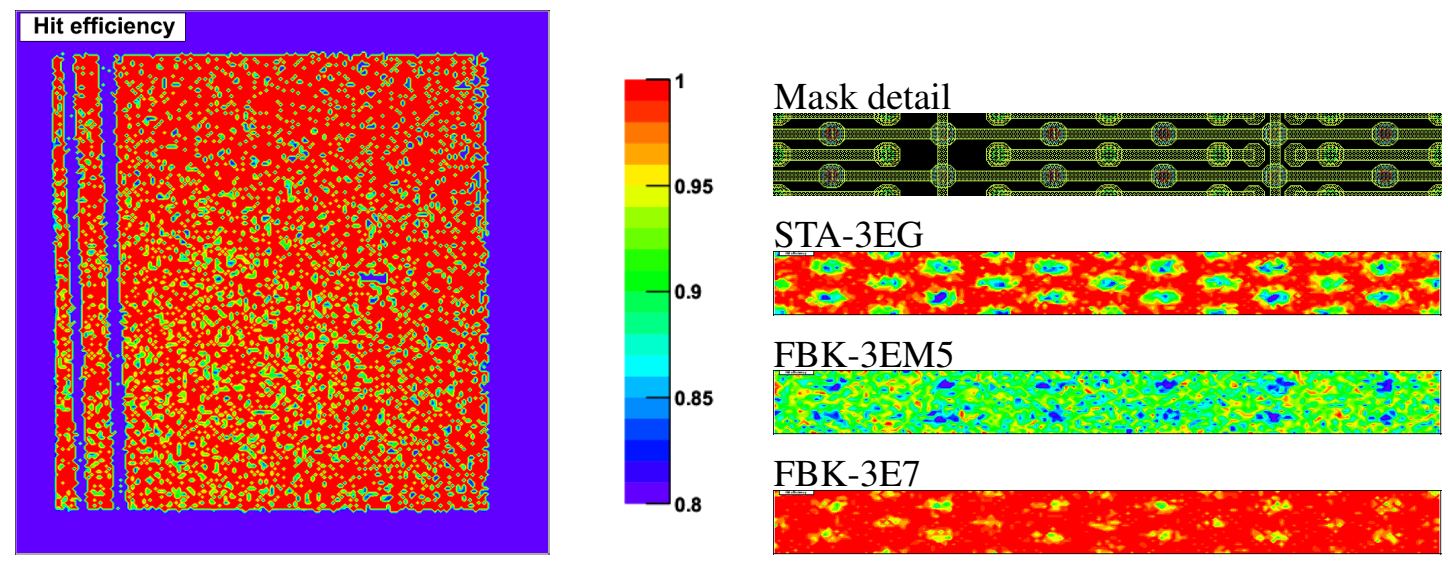

Figure 3: Hit efficiency at normal incidence. The left-hand plot shows the overall response for the STA-3EG sensor, the horizontal and vertical lines are artefacts due to masked strips in the telescope. The right-hand column of plots show the efficiency of the various sensors, superimposed into a $2 \times 2$ cell as illustrated by the mask detail.

\subsection{Charge sharing}

Charge sharing in the ATLAS pixel geometry happens predominantly along the long ( $400 \mu \mathrm{m})$ edge of the pixels. Figure 4 shows the charge sharing probability as a function of the distance from the pixel edge. For tracks of normal incidence, the magnetic field in general cause an enhancement 
in the charge sharing but the effect in the 3D devices is much less pronounced than in the planar device. For inclined tracks the charge sharing diminish with magnetic field, but again only a small response is seen in the $3 \mathrm{D}$ sensors.
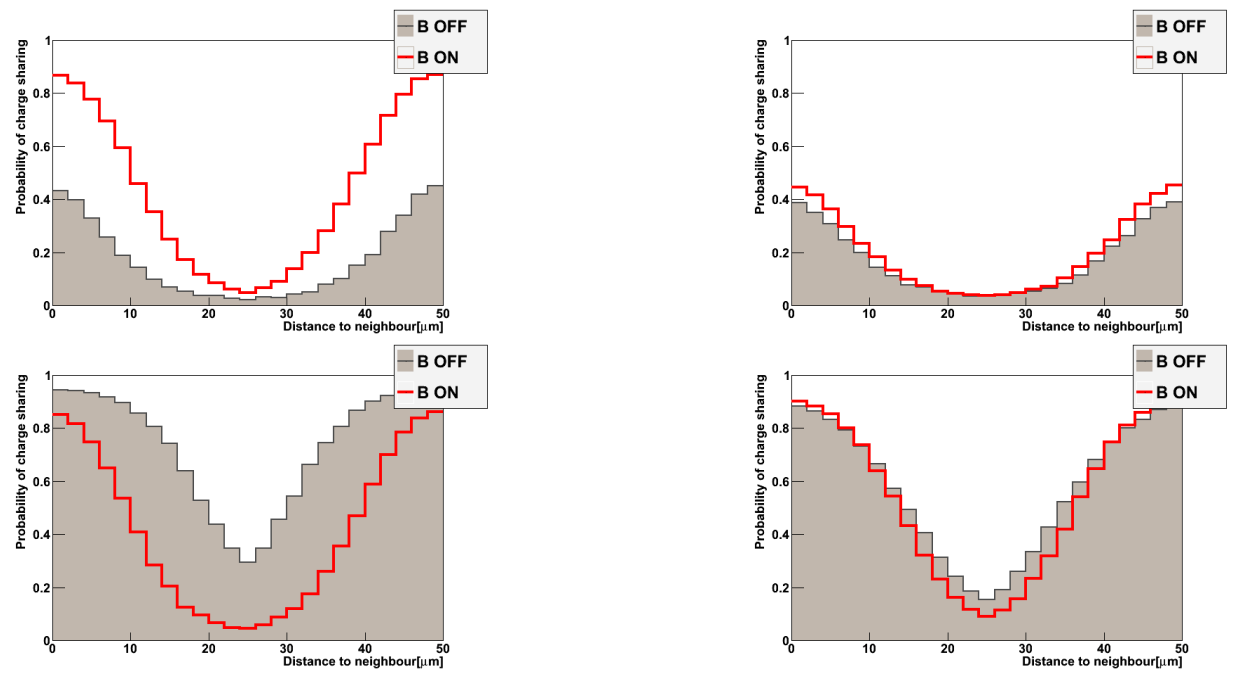

(a) Planar

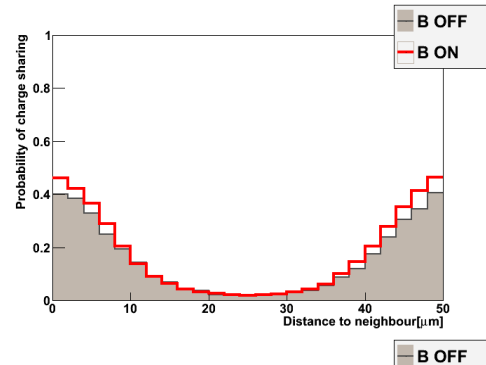

(b) STA-3EG
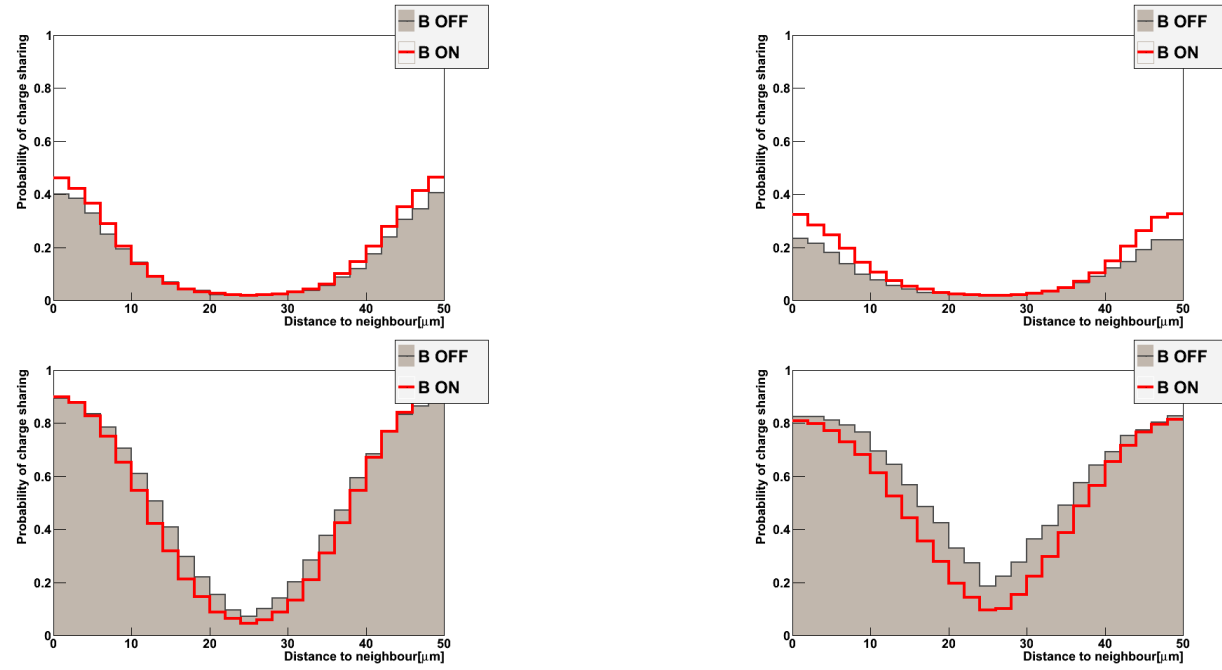

(c) FBK-3E7

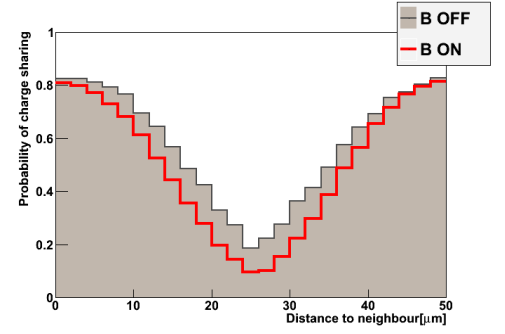

(d) FBK-3EM5

Figure 4: Charge sharing probability as a function of the distance from the long pixel edge. The upper row shows normal incidence tracks, the lower row shows tracks with inclined incidence.

\subsection{Position resolution}

For tracks where the charge is shared between two neighboring pixels the hit position is estimated using charge-weighted position $\eta=\left(q_{1} x_{1}+q_{2} x_{2}\right) /\left(q_{1}+q_{2}\right)$, subsequently corrected using the observed distribution $\mathrm{d} \eta / \mathrm{d} \eta$, employing an approach similar to [14]. The resulting track residuals for all cluster sizes are shown in Figure 5. The observed resolution follows closely the tendency to charge sharing. 

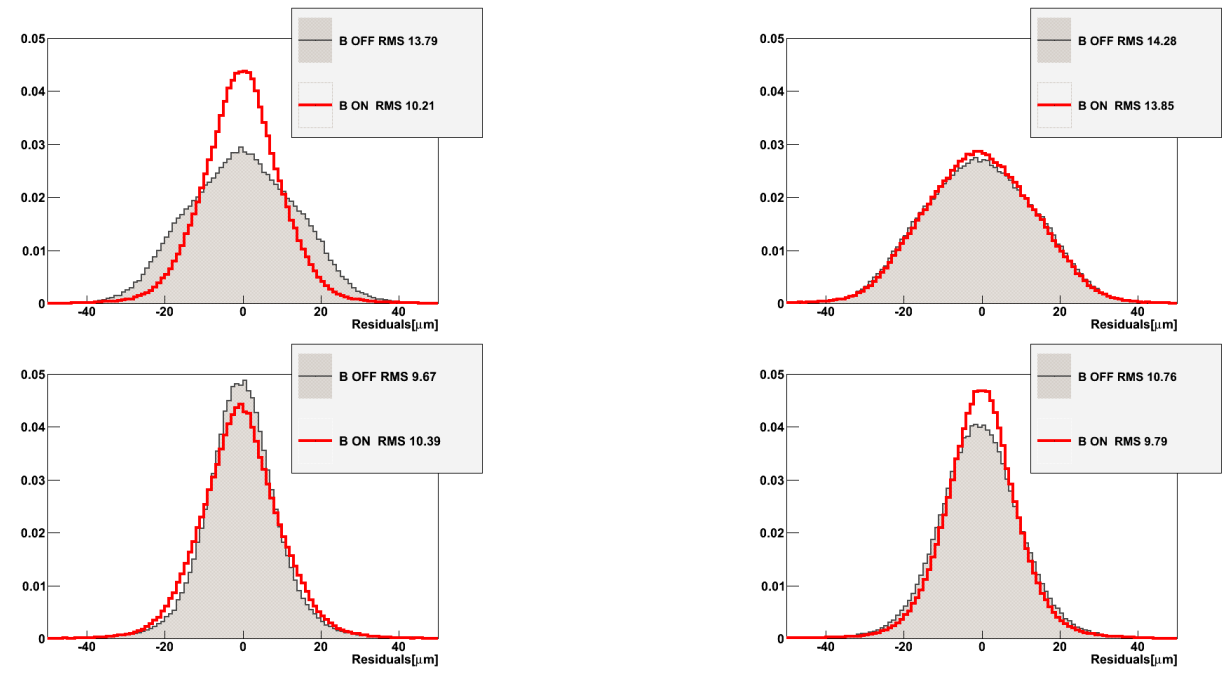

(a) Planar

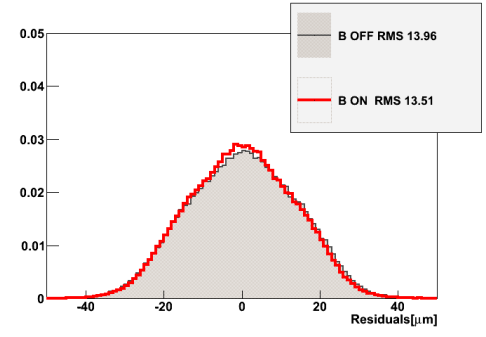

(b) STA-3EG

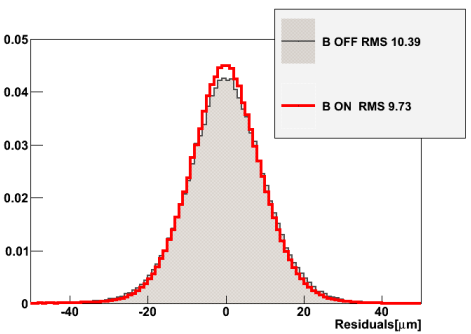

(c) FBK-3E7
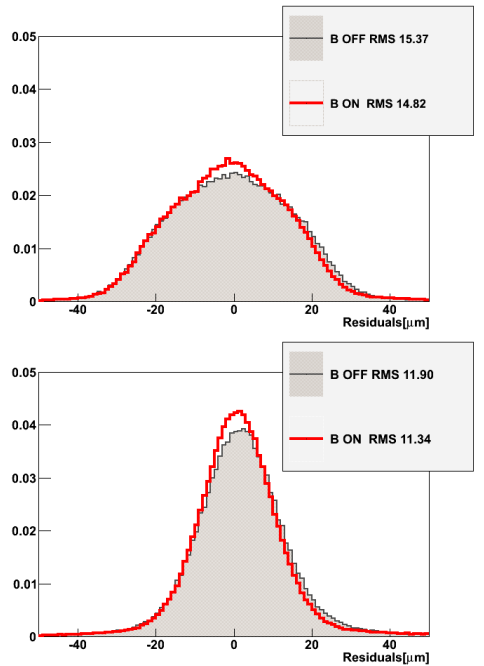

(d) FBK-3EM5

Figure 5: Residual distributions between the extrapolated track and the corrected charge-weighted hit position. The upper row shows normal incidence tracks, the lower row shows tracks with inclined incidence.

\section{Conclusion and outlook}

It has been shown that the cluster size of charged track hits in 3D pixel sensors is only weakly affected by a magnetic field of $1.6 \mathrm{~T}$. Important operational parameters like over-all efficiency and resolution are largely determined by the track incidence angle, and the performance above about $12^{\circ}$ is close to that of a planar sensor.

In order to resolve the pixel response map to more detail, the ATLAS 3D R\&D collaboration has already taken data with a higher resolution telescope. Additionally, the magnetic field data sets have also been supplemented with runs at a wide range of incidence angles. The analysis of these data is currently in progress. Future test beam efforts by the current authors will focus on the characterization of 3D devices irradiated in excess of $10^{15} \mathrm{n} / \mathrm{cm}^{2}$. 


\section{References}

[1] Sherwood I. Parker, Christopher J. Kenney, and Julie Segal. 3-D: A New architecture for solid state radiation detectors. Nucl. Instrum. Meth., A395:328-343, 1997. doi:10.1016/S0168-9002(97)00694-3

[2] C Kenney, S Parker, J Segal, and C Storment. Silicon detectors with 3-D electrode arrays: Fabrication and initial test results. IEEE Trans. Nucl. Sci., 46:1224-1236, 1999. doi:10.1109/23.785737

[3] C.J. Kenney, S. Parker, and E. Walckiers. Results from 3-d silicon sensors with wall electrodes: near-cell-edge sensitivity measurements as a preview of active-edge sensors. IEEE Trans. Nucl. Sci., 48(6):2405-2410, Dec 2001. doi:10.1109/23.983250

[4] C.J. Kenney, J.D. Segal, E. Westbrook, Sherwood Parker, J. Hasi, C. Da Vià, S. Watts, and J. Morse. Active-edge planar radiation sensors. "Nucl. Instrum. Meth.", A565:272-277, 2006. doi:10.1016/j.nima.2006.05.012

[5] S.I. Parker and C.J. Kenney. Performance of 3-d architecture silicon sensors after intense proton irradiation. IEEE Trans. Nucl. Sci., 48(5):1629-1638, Oct 2001. doi:10.1109/23.960351

[6] Sherwood Parker, G Anelli, C Da Vià, J Hasi, Pierre Jarron, C Kenney, A Kok, E Perozziello, and S J Watts. Advances in silicon detectors for particle tracking in extreme radiation environments. Nucl. Instrum. Meth., A509:86-91, 2003. doi:10.1016/S0168-9002(03)01554-7.

[7] C. Da Vià et al. Radiation hardness properties of full-3D active edge silicon sensors. Nucl. Instrum. Meth., A587:243-249, 2008. doi:10.1016/j.nima.2007.12.027

[8] C. Da Vià, E. Bolle, K. Einsweiler, M. Garcia-Sciveres, J. Hasi, C. Kenney, V. Linhart, Sherwood Parker, S. Pospisil, O. Rohne, T. Slavicek, S. Watts, and N. Wermes. 3D active edge silicon sensors with different electrode configurations: Radiation hardness and noise performance. Nucl. Instrum. Meth., A604(3):505 - 511, 2009. doi:10.1016/j.nima.2009.03.049

[9] G Aad et al. Atlas pixel detector electronics and sensors. Journal of Instrum., 3(07):P07007, 2008. doi:10.1088/1748-0221/3/07/P07007

[10] Ivan Peric, Laurent Blanquart, Giacomo Comes, Peter Denes, Kevin Einsweiler, Peter Fischer, Emanuele Mandelli, and Gerrit Meddeler. The FEI3 readout chip for the ATLAS pixel detector. Nucl. Instrum. Meth., A565(1):178 - 187, 2006. doi:10.1016/j.nima.2006.05.032.

[11] G. F. Dalla Betta et al. Characterization of 3d-ddtc detectors on p-type substrates. arXiv, 2009. arXiv:0911.4864

[12] M. Mathes et al. Test beam characterizations of 3D silicon pixel detectors. IEEE Trans. Nucl. Sci., 55:3731-3735, 2008. doi:10.1109/TNS.2008.2005630

[13] J. Treis et al. A modular PC based silicon microstrip beam telescope with high speed data acquisition. Nucl. Instrum. Meth., A490:112-123, 2002. doi:10.1016/S0168-9002(02)00913-0

[14] I. Gorelov et al. A measurement of Lorentz angle and spatial resolution of radiation hard silicon pixel sensors. Nucl. Instrum. Meth., A481:204-221, 2002.

doi:10.1016/S0168-9002(01)01413-9 